\title{
Trimethoprim Sulfamethoxazole - Induced Drug Reaction Syndrome with Eosinophilia and Systemic Symptoms (DRESS)
}

\author{
Stephanie $\mathrm{P}^{1 *}$, Saturno $\mathrm{B}^{2}$ and Rosanelly $\mathbf{R}^{3}$ \\ ${ }^{1}$ Doctor of Medicine, Central University of Venezuela, Venezuela \\ ${ }^{2}$ Specialist in Internal Medicine, University Hospital of Caracas, Central University of Venezuela, \\ Venezuela \\ ${ }^{3}$ Specialist in Dermatology and Internal Medicine, Instructor Professor, Military Hospital Dr. \\ Carlos Arvelo, Central University of Venezuela, Venezuela
}

Case Report

Volume 5 Issue 4

Received Date: October 22, 2020

Published Date: November 13, 2020

DOI: $10.23880 /$ cdoaj-16000223

*Corresponding author: Stephanie Plaza, Central University of Venezuela. El Incario St, 284, Santiago de Surco, Lima, Peru, Tel: +51997129064; Email: Stephanie.plaza@hotmail.com

\section{Abstract}

Drug reaction with eosinophilia and systemic symptoms syndrome (DRESS) is a pharmacological hypersensitivity reaction, most frequently associated with antiepileptic drugs and sulfonamides, characterized by skin rash, fever, lymphadenopathy, facial edema, hematological alterations and visceral organ involvement, which generally present within 8 weeks from the beginning of treatment. The mortality rate is $10-20 \%$ and liver failure represents the main cause of death in these patients. We report a case of a 24-year-old female patient with a history of inflammatory acne under treatment with trimethoprim sulfamethoxazole (tab 800/160 mg every 12 hours) who presented, on her third week of treatment, a skin rash, fever, facial edema, lymphadenopathy, eosinophilia and liver failure. The drug was discontinued and treatment with intravenous hydrocortisone at a therapeutic dose was started, observing a decrease in liver enzymes after 4 days of treatment, with a good outcome.

Keywords: DRESS; Drug; Trimethoprim sulfamethoxazole

\section{Introduction}

In 1996 Bocquet described for the first time a hypersensitivity syndrome that began in the first 2 months after the introduction of a drug; it was characterized by severe skin involvement, facial edema, fever, enlarged lymph nodes, hematological abnormalities (eosinophilia and atypical lymphocytes) and internal organ involvement (hepatitis, carditis, interstitial nephritis or interstitial pneumonitis); he proposed the name of pharmacological reaction with eosinophilia and systemic symptoms (DRESS) [1]. The incidence of DRESS is estimated between 1: 1,000 to $1: 10,000$ drug exposures, it is observed more frequently in adults, and the mortality rate is high, from 10 to $20 \%$ [2].
Aromatic anticonvulsants and sulfonamides are the most frequent causative agents [3].

The pathophysiological mechanism by which DRESS syndrome occurs is unknown, but three mechanisms have been suggested:

i. Genetic components that alter the patient's immune response.

ii. Reactivation of a viral infection by Herpes Virus type 6-7, Epstein Barr Virus or Cytomegalovirus.

iii. Defect in drug metabolism resulting in decreased elimination of intermediate metabolites [2].

The disease usually begins, 2 to 3 weeks after the 
introduction of the triggering drug, with an erythematous morbilliform maculopapular rash, in most cases, pruritic, and fever $>38^{\circ} \mathrm{C}$. The skin rash may be maculopapular, pustular, or urticarial, and purpuric lesions that, in some instances, may converge to form plaques. Typically involved sites are the face, trunk, and extremities. It is characteristic to find, in the initial phase of the disease, facial and periorbital edema. The mucosal surfaces, palms and soles are usually not affected. It is typical to find lymphadenopathy in the cervical, axillary or inguinal region [4].

The liver is the organ most frequently affected and liver failure represents the main cause of death in these patients. In $11 \%$ of cases there is kidney involvement, with elevated urea, creatinine and proteinuria; some patients present with adult respiratory distress syndrome and interstitial pneumonitis. Neurologic complications include meningitis, cerebral edema, and cranial nerve involvement. Myocarditis is a rare and fatal manifestation in DRESS [2].

Treatment is based on early withdrawal of the drug [5]. Systemic corticosteroids are the first line of treatment, improving clinical symptoms in the acute phase. In severe cases, the use of intravenous immunoglobulin (IVIG) is recommended [4]. The French Society of Dermatology recommends the use of ganciclovir in patients with confirmation of a major viral reactivation of HHV-6 [2].

\section{Case Report}

We present a 24-year-old female patient who started illness 10 days prior to admission, with a widespread erythematous and itchy rash, that appeared after sunlight exposure, and partially improved with the intake of oral antihistamines at therapeutic doses. Later she presented fever, asthenia, myalgia and holocranial headache of moderate intensity accompanied by 2 emesis. Her medical record was notable for a history of inflammatory acne currently in her third week of treatment with trimethoprim sulfamethoxazole (tab 800/160 mg, orally, every 12 hours). On physical examination, the patient had a confluent erythematous maculopapular rash, involving trunk and lower limbs, sparing palms of hands and feet, oral and genital mucosa (Figures 1-5); she also presented bipalpebral edema and cervical lymphadenopathies (Figure 6). Laboratory study results are summarized in Table 1. Serology for Epstein Barr, Cytomegalovirus, Dengue and Herpes simplex result was negative. Sulfamethoxazole-trimethoprim was withdrawn. A skin biopsy revealed severe dermatitis changes associated with perivascular and perianexial lymphocytic infiltration, spongiosis of cells of the epidermis and mild edema of the papillary dermis, suggestive of early stages of dermatitis due to drug reactions. The diagnosis of DRESS syndrome was made and treatment with Hydrocortisone (100mg, intravenously, every 12 hours), Chlorpheniramine (10mg, intravenously, every 8 hours) and Acyclovir (400mg, intravenously, every 8 hours), was begun, with successful resolution of initial clinical symptoms and improvement of liver function. She was discharged on the fourth day of admission, with successive outpatient controls. After two weeks there was a complete remission of skin changes (Figures 7-10).

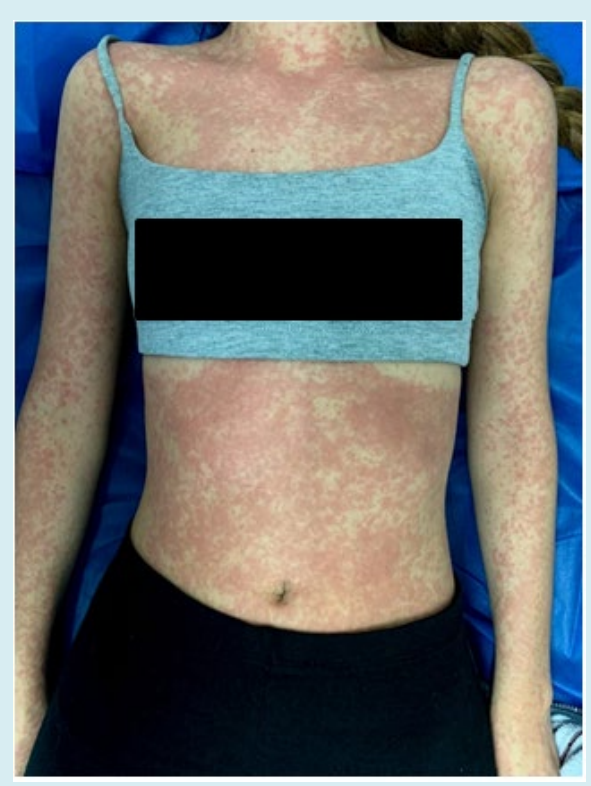

Figure 1: Erythematous maculopapular rash.

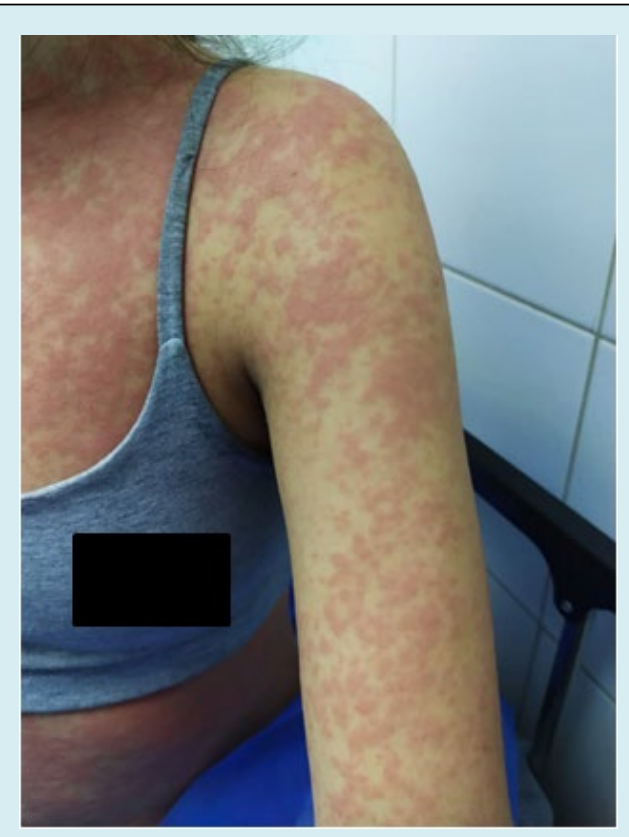

Figure 2: Erythematous maculopapular rash. 


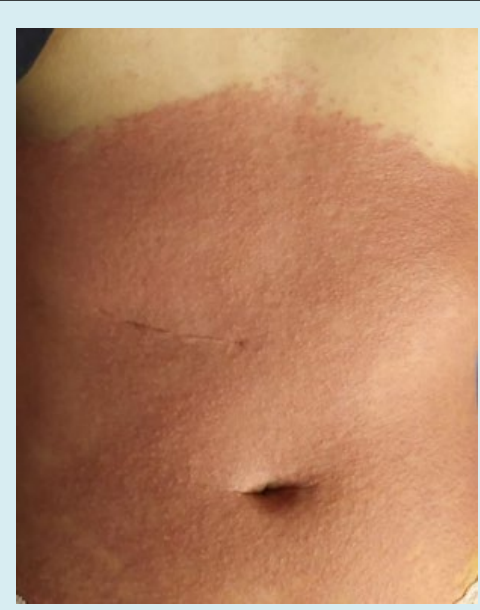

Figure 3: Confluent erythematous maculopapular rash.

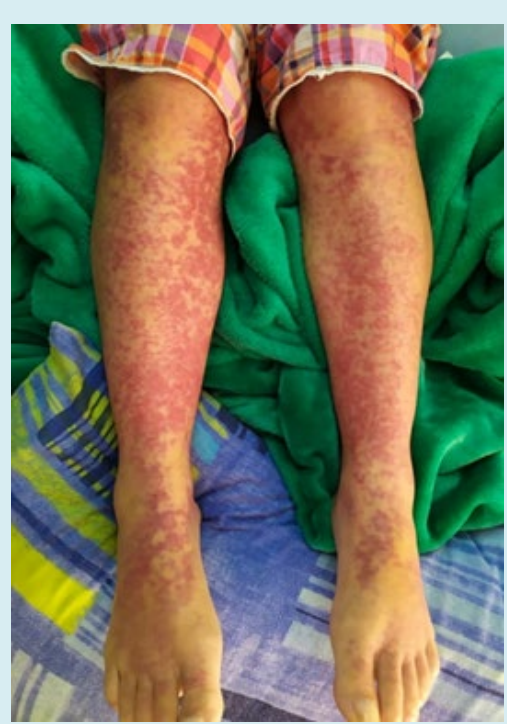

Figure 4: Confluent erythematous maculopapular rash in both legs.

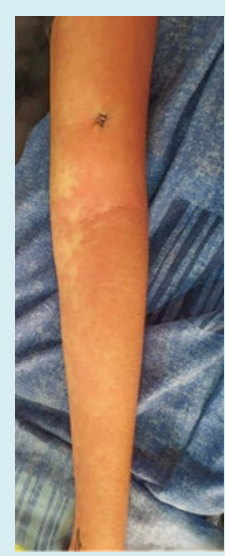

Figure 5: Confluent erythematous maculopapular rash in left arm.
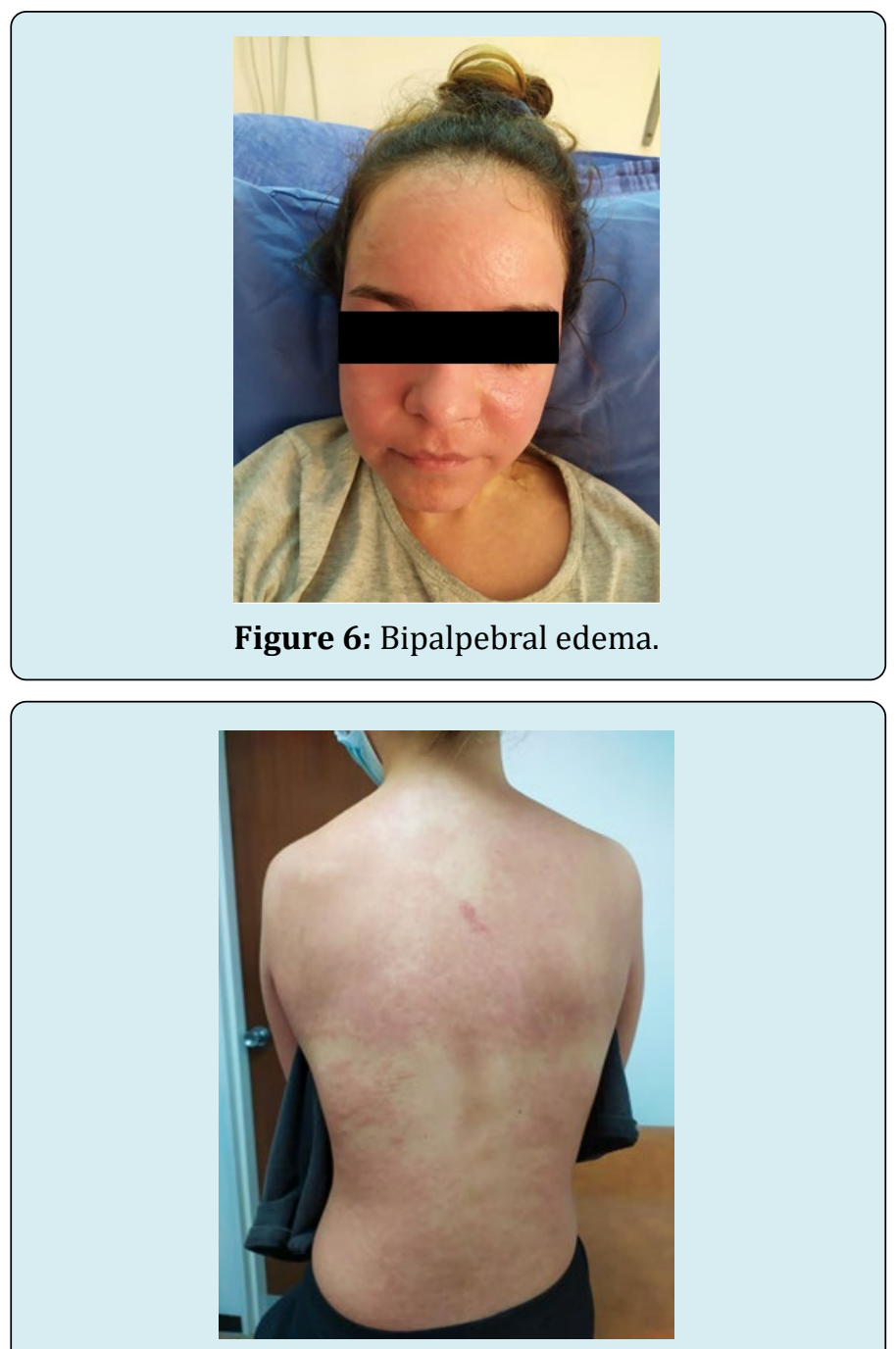

Figure 7: Confluent erythematous maculopapular rash.

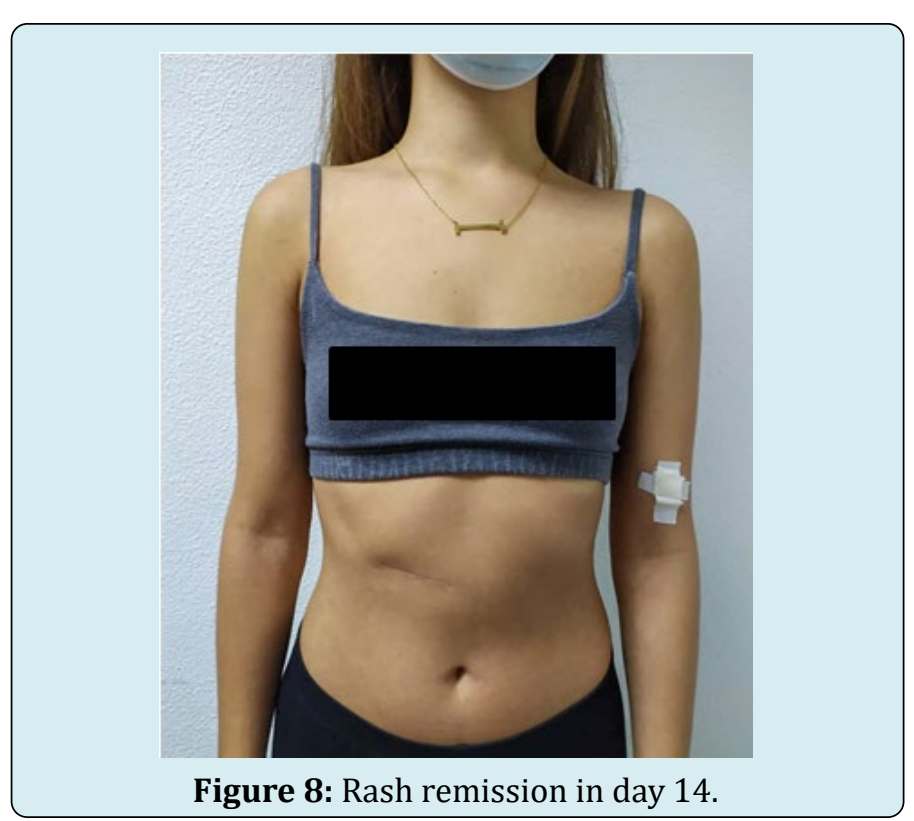




\section{Clinical Dermatology Open Access Journal}
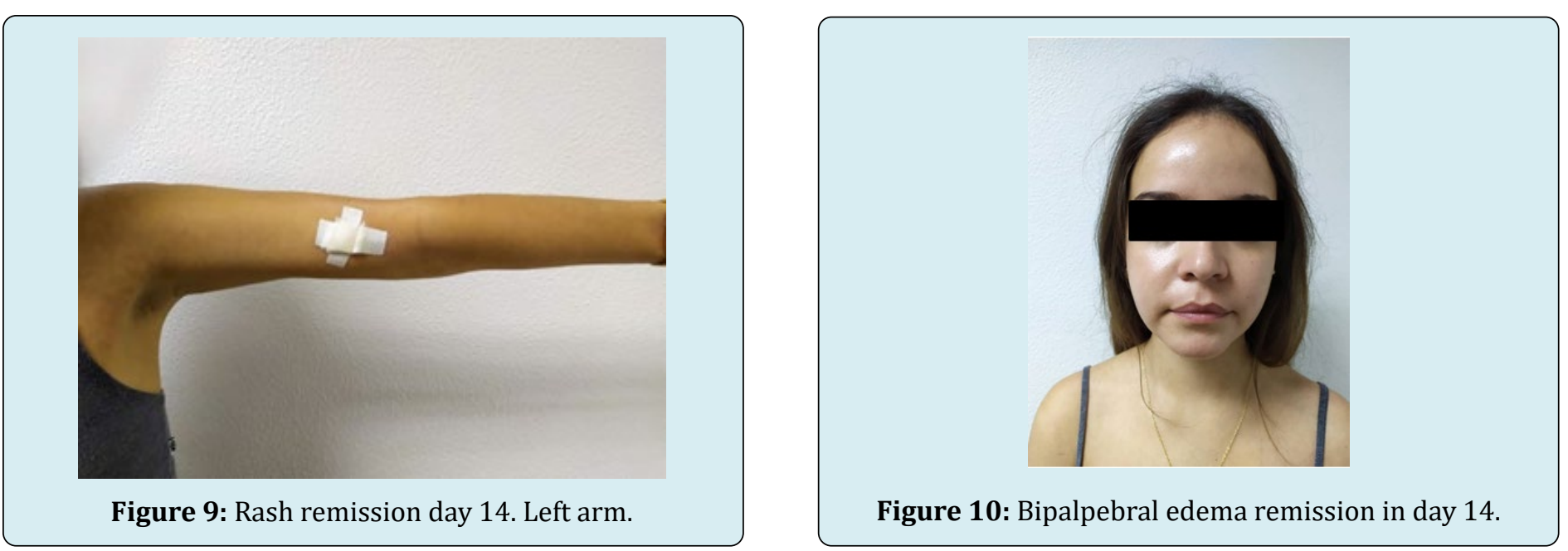

\begin{tabular}{|c|c|c|}
\hline $\begin{array}{c}\text { Admission day } \\
\text { (month - day - year) }\end{array}$ & $\begin{array}{c}\text { Day } 1 \text { of hospitalization } \\
9.05 .20 \\
\end{array}$ & $\begin{array}{c}\text { Day } 2 \text { of hospitalization } \\
9.06 .20 \\
\end{array}$ \\
\hline Leukocytes $(\mathrm{cel} / \mu \mathrm{L})$ & 10400 & 10600 \\
\hline Lymphocytes \% & 23 & 38.1 \\
\hline Neutrophils \% & 66 & 39.8 \\
\hline Eosinophils \% & 6 & 10.8 \\
\hline Hemoglobin $(\mathrm{g} / \mathrm{dl})$ & 12.8 & 10.9 \\
\hline MCV(fL) & 85 & 76.9 \\
\hline HCM & 27.8 & 26 \\
\hline ALT (UI/L) & 491 & 434 \\
\hline AST (UI/L) & 216 & 137 \\
\hline Total bilirubin (mg/dl) & 1.17 & 0.64 \\
\hline Direct bilirubin & 0.89 & 0.33 \\
\hline Indirect bilirubin & 0.28 & 0.31 \\
\hline Albumin $(\mathrm{g} / \mathrm{dl})$ & 3.3 & 2.9 \\
\hline Alkaline phosphatase (UI/L) & 218 & 168 \\
\hline LDH (UI/L) & 824 & 584 \\
\hline GGT (UI/L) & 270 & 216 \\
\hline $\mathrm{C}$ - reactive protein & 1.1 & 1 \\
\hline Erythrocyte sedimentation rate $(\mathrm{mm})$ & 2 & 2 \\
\hline Creatinine (mg/dl) & 0.68 & \\
\hline PT (patient) sec & 13.2 & 11.6 \\
\hline PT (control) sec & 12.3 & 12.3 \\
\hline Reason & 1.07 & 0.94 \\
\hline INR & 1.06 & 0.95 \\
\hline
\end{tabular}

Table 1: Laboratory study results. 


\section{Clinical Dermatology Open Access Journal}

\section{Discussion}

Our patient was diagnosed as DRESS syndrome as defined by Bocquet, et al. [1]. The clinical features were typical: fever, lymphadenopathy, rash, hypereosinophilia, and liver involvement. According to the RegiSCAR scoring system established by Kardaun, et al. (Table 2) [6], added 6 points, considering it as a definitive diagnosis.

\begin{tabular}{|c|c|c|c|}
\hline Features & NO & YES & UNKNOWN \\
\hline Fever $\left(>38^{\circ} \mathrm{C}\right)$ & -1 & 0 & -1 \\
\hline $\begin{array}{l}\text { Enlarged lymph nodes } \\
(>2 \text { sites } />1 \mathrm{~cm})\end{array}$ & 0 & 1 & 0 \\
\hline Atypical Lymphocytes & 0 & 1 & 0 \\
\hline Eosinophilia & \multirow{3}{*}{0} & 0 & \multirow{3}{*}{0} \\
\hline $10 \%-19.9 \%$ & & 1 & \\
\hline$>20 \%$ & & 2 & \\
\hline Skin rash & 0 & & 0 \\
\hline Extent $>50 \%$ & 0 & 1 & 0 \\
\hline Edema, infiltration, purpura, scaling & -1 & 1 & 0 \\
\hline Biopsy suggesting DRESS & -1 & 0 & 0 \\
\hline Internal organ involvement & \multirow{3}{*}{0} & & \multirow{3}{*}{0} \\
\hline One & & 1 & \\
\hline Two or more & & 2 & \\
\hline Resolution in $>15$ days & $1-$ & 0 & -1 \\
\hline
\end{tabular}

Table 2: RegiSCAR Diagnosis Score for DRESS.

The association of DRESS syndrome with sulfonamides occurs quite frequently, similar conditions have been described since 1959 [1]. The patient's symptoms began 20 days after starting the drug and completely improved 2 weeks after its withdrawal. At the time of admission, the triggering drug was immediately suspended and early treatment with corticosteroids was begun. Due to the possibility of viral reactivation by HHV-6, treatment with intravenous acyclovir was started, which was omitted after obtaining negative serology for EB, CMV and HHV-6.

In Taiwan, a retrospective study of 60 cases showed that the mean latency period of the drug reaction for all patients was 20.7 days (range, 3-76 days). The most common symptom was fever (52 patients [87\%]) and diffuse rash (60 patients [100\%]), some with facial edema. Elevation of liver enzymes was the most common finding related to systemic involvement (48 of 60 [80\%]), however, only 4 cases progressed to liver failure, and most of them subsequently resolved. Forty-five of the 60 patients $(75 \%)$ were treated with systemic corticosteroids, of which 5 died. In 2 patients, IVIG was administered, 1 died. Mortality was 10\% [7].

DRESS syndrome is a life threatening multisystem adverse drug reaction that must be recognized promptly. The outcome and prognosis will be better when early recognition, causative drug withdrawn and establishment of supportive treatment. Organic involvement is potentially serious; liver damage is the most common (64\%) and causes most of the mortality associated with this syndrome. The hepatobiliary symptoms vary from a mild elevation of liver enzymes to fulminant hepatitis that leads to the death of patients in a non-negligible percentage of cases [8].

\section{References}

1. Bocquet H, Bagot M, Roujeau JC (1996) Drug-induced pseudolymphoma and drug hypersensitivity syndrome (drug rash with eosinophilia and systemic symptomsDRESS). Semin Cutan Med Surg 15(4): 250-257.

2. Waseem D, Muzamil L, Najeebullah S, Imtiyaz D, Qayoom $\mathrm{K}$, et al. (2016) Dress Syndrome: A Review and Update. Imed pub 1(1): 1-8.

3. María Mercedes FR, Arturo Ramírez R, Israel Guerrero I, Alicia López R, et al. (2018) DRESS syndrome. Dermatol Rev 62(6): 522-528. 
4. Shiohara T, Mizukawa Y (2019) Drug-induced hypersensitivity syndrome (DiHS)/drug reaction with eosinophilia and systemic symptoms (DRESS). Allergol Int 68(3): 301-308.

5. Lee JH, Park HK, Heo J, Kim TO, Kim GH, et al. (2018) Drug Rash with Eosinophilia and Systemic Symptoms (DRESS) syndrome Induced by Celecoxib and Antituberculosis Drugs. J Korean Med Sci 23(3): 521-525.

6. Pannu A, Saroch A (2017) Diagnostic criteria for drug rash and eosinophilia with systemic symptoms. J Family Med Prim Care Rev 6(3): 693-694.

7. Chen YC, Chiu HC, Chu CY (2010) Drug Reaction With Eosinophilia and Systemic Symptoms A Retrospective Study of 60 Cases. Arch Dermatol 146(12): 1373-1379.

8. Casal-Beloy I, García-Novoa MA, García-González M, Rey Simó I (2019) DRESS syndrome, a differencial diagnosis in late postoperative complications. An Sist Sanit Navar 42(1): 89-92. 